\title{
Load analysis and deformation research of the flexible bearing based on a three-force ring superposition method
}

\author{
Yang $\mathrm{Yu}^{1, *}$, En $\mathrm{Zhu}^{1}$, Xiaoyang $\mathrm{Chen}^{1}$, and Yang Wang ${ }^{2}$ \\ ${ }^{1}$ School of Mechatronic Engineering and Automation, Shanghai University, Shanghai 200444, PR China \\ 2 Beijing CTKM Harmonic Drive Co., Ltd, Beijing 101318, PR China
}

Received: 1 February 2021 / Accepted: 5 April 2021

\begin{abstract}
The flexible bearing is a key component of harmonic reducer enabling the flexspline to generate a controllable elastic deformation. Its performance and life will significantly affect the normal operation of harmonic reducer. In order to improve the life and the working performance of the flexible bearing, the calculation of its load distribution is necessary. Based on the theory of thin-walled ring, the relationship between radial deformation and load of three-force ring was derived. Then a superposition model of three-force ring was developed to calculate rapidly the load distribution of flexible bearings with elliptical cams in harmonic reducers. The validity of the proposed model was proved by comparing with a static analysis model. In this paper, the influences of ball number and external load on the load distribution, radial deformation and bending normal stress of outer ring were investigated by three-force ring superposition method. Based on the deformation of three-force ring, the deformation characteristics and bending normal stress distribution of outer ring of the flexible bearing were analyzed, and several regular results were found.
\end{abstract}

Keywords: Load analysis model / flexible bearing / load distribution / stress calculation / radial deformation

\section{Introduction}

Harmonic reducer is widely used in aerospace, medical devices, robots, CNC machine tools, packaging equipment, instruments and other fields. As one of the main working components, the flexible bearing (FB)'s damage is one of the main failure reasons of harmonic drive [1-3]. It is necessary to study the load distribution in flexible bearing for high efficiency, high precision and long life of harmonic drive.

The harmonic reducer is mainly formed of three components: circular spline (CS), flexspline (FS) and wave generator (WG), where the WG consists of a FB and a wave generator cam (WG cam), shown in Figure 1. When the CS is fixed and the WG is the driving member, the FS becomes the follower. The WG enables the FS to generate a controllable elastic deformation, it forces that the teeth at both ends of the major axis of the CS and the FS are in full engagement, while the teeth at both ends of the minor axis are completely detached. When the WG rotates, the teeth of the FS mesh with the teeth of the CS in turn, repeating four states: engaging-in, engaging, engaging-out and disengaging, shown in Figure 1. Thus, this staggered tooth

\footnotetext{
* e-mail: yy19960210@163.com
}

transmission makes the harmonic reducer drive with great deceleration ratio.

The FB is of great difference compared with the ordinary bearing. In structure, the thickness of FB is much smaller than that of ordinary bearing. After the FB is assembled on the WG cam, the inner and outer rings will have a certain deflection, and the shape depends on that of the WG cam. In loading form, when the harmonic reducer works, the flexible bearing will bear symmetrical external load at both ends of the major axis. Even there is no external load, the internal load distribution of FB is quite different from that of ordinary bearing due to the predeformation caused by WG cam.

The calculation and research of load distribution in rolling bearing is the analysis basis of kinematics, lubrication mechanics, contact fatigue life and efficiency of rolling bearing, which is necessary for improving the life and the working performance of rolling bearing. For the ordinary rolling bearing, the analysis and calculation of its load distribution has become the mature theory $[4,5]$. However, the deformation, load and failure modes of the FB are quite different from those of ordinary bearing. Thus, it isn't accurate to study the FB by the existing theory. In order to research the mechanical characteristics of the FB profoundly, the primary step is to obtain its exact internal load distribution. 


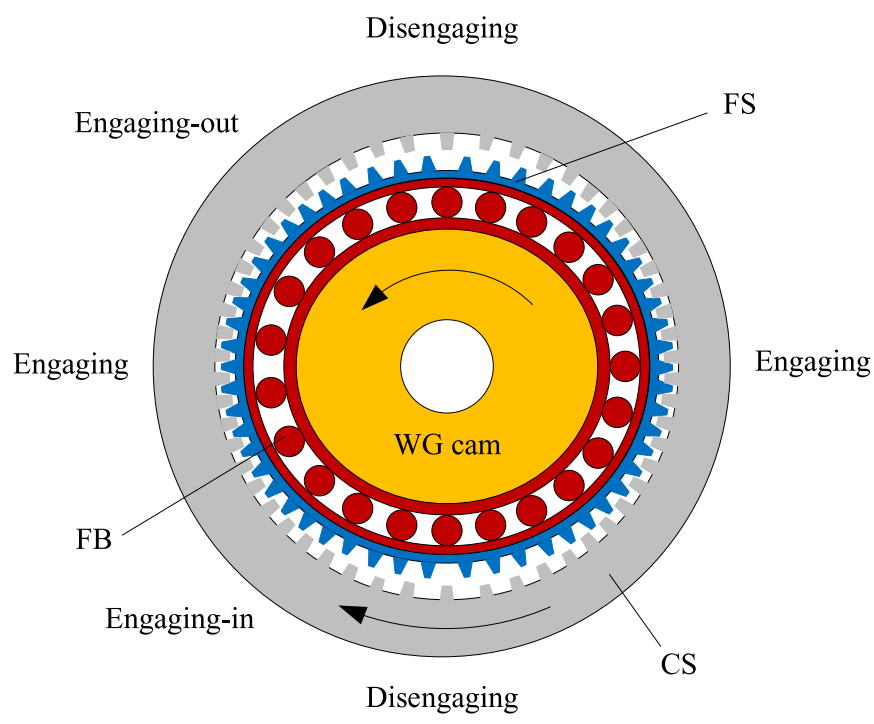

Fig. 1. Schematic diagram of the working principle of the harmonic reducer.

Several theoretical methods were applied for studying the load distribution in the FB. Le and Shen [6] obtained the load distribution of the FB using the curved beam theory of elasticity and material mechanics, three-moment theory of continuous beam, and energy method theory. The load decomposition obtained by the model is in good agreement with the experimental results. But this method can only calculate the bearing load distribution of the FB mounted on a four-force action type cam. The deflection of the outer ring of the FB was calculated based on the circular radial deformation under a static equilibrium state and Shao and Wen [7] obtained the load on the rolling element by solving a set of nonlinear deformation coordination equations. However, only the case of odd number balls was discussed in the model. A single-force thin-walled ring superposition model was established to calculate the load distribution of the FB fitted on a cam $[8,9]$. This method could be used to calculate the load distribution in the FB with random number of balls, but the external loads are not considered in this model. And a two-force thin-walled ring super-position model was established to calculate the load distribution of the FB fitted on a cam [10]. However, this method could only be applied for the FB with even number of balls. Therefore, it is necessary to provide a new theoretical method for calculating the load distribution in the FB with random number of balls, which is in force balance at the same time.

According to the general solution of the deformation of the thin-walled ring deduced by Liu and Chiu [11], Xiong et al. [12] developed a universal static analysis model to analyze the load distribution of different FBs in the harmonic reducer. Not only arbitrary numbers of balls (even or odd numbers) can be considered in this model but also arbitrary radial symmetric external loads.

Apart from theoretical analysis, finite element methods (FEMs) were also employed in the load analysis of the FB $[13,14]$. Compared with the theoretical methods, this kind of method requires a lot of computing time.
In this paper, a superposition model of three forces ring was proposed based on the theory of thin-walled ring and superposition principle. According to this mechanical model, the internal load distribution in the FB could be calculated. And then the radial deformation and the bending normal stress on the outer ring were also calculated and analyzed. Based on the calculation of this mechanical model, we investigated the influences of the number of balls, the angle of the ball position and the load torque on the ball load. And the influences of the numbers of balls and the load torque on the radial deformation and maximum bending normal stress of outer ring were subsequently studied.

\section{A mechanical model of three-force ring}

\subsection{The basic theory}

A ring whose cross-section size is much smaller than its radius is called a thin-walled ring. It is assumed that the cross-section of the ring is rectangular and the shape is unchanged along the circumference. And the load is uniformly distributed along the width of the ring. Under these conditions, stress and displacement are constant along the width, so it can be treated as a plane problem. For the simplification of calculation, the mechanical model has the following basic assumptions: (1) It is assumed that the load distribution inside the FB is equal to that the outer ring bears multiple radial loads, as shown in Figure 2. (2) It is assumed that the deformation of the outer ring is small, so the methods of material mechanics and superposition principle could be used to study the load distribution of outer ring.

In this paper, three main formulas about the theory of thin-walled ring $[15,16]$ are given by:

$$
\begin{gathered}
\frac{d^{2} w}{d \varphi^{2}}+w=-\frac{M R^{2}}{E I} \\
w d \varphi+d v=0 \\
\theta=\left(\frac{1}{R}\right)\left(w-\frac{d v}{d \varphi}\right)
\end{gathered}
$$

Equation (1) is the elastic equation connecting bending moment $M$ and radial displacement $w$, where $E$ is the elastic modulus of the material and $I$ is the inertial moment of the ring section. Equation (2) is derived from no elongation hypothesis, and it indicates the relationship between radial displacement $w$ and circumferential displacement $v$. Equation (3) is the expression of normal angle $\theta$ of circular section.

The deformation of outer ring of flexible bearing can be analyzed by the theory of thin-walled ring because of its small thick-ness. In the following analysis and calculations, the shape of the ring is indicated by the neutral layer curve of the ring, and the load and deformation of points on the neutral layer are discussed. 


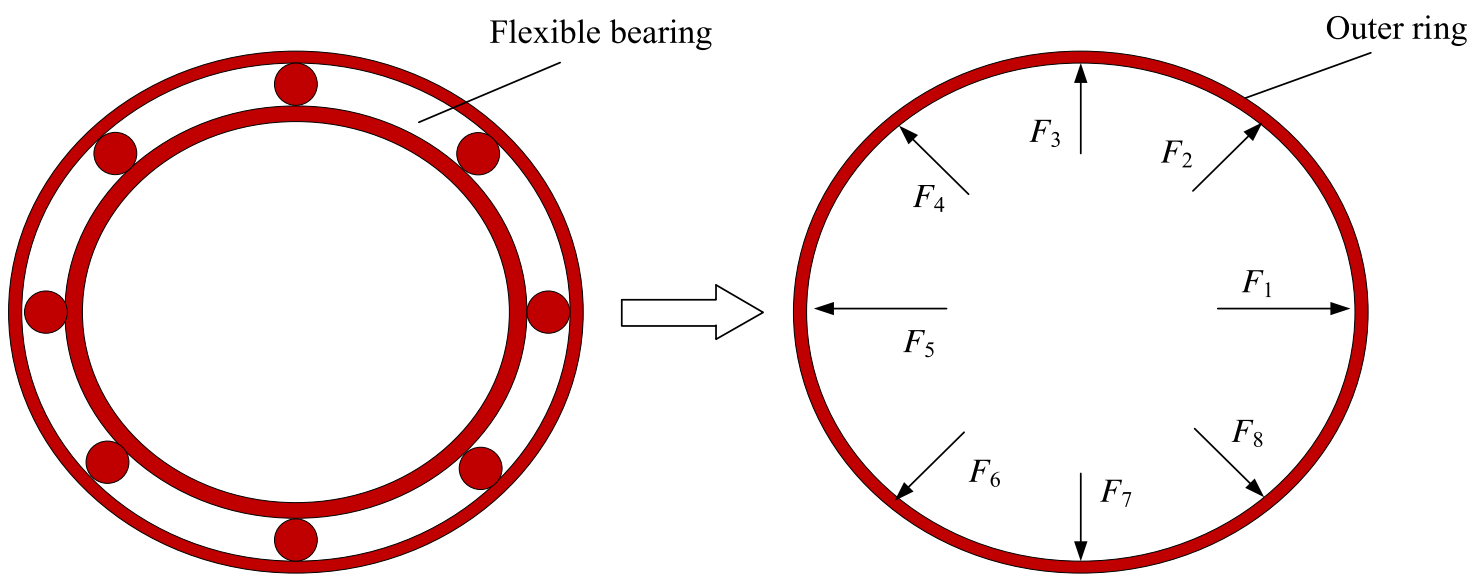

Fig. 2. Load simplification of outer ring of the FB.

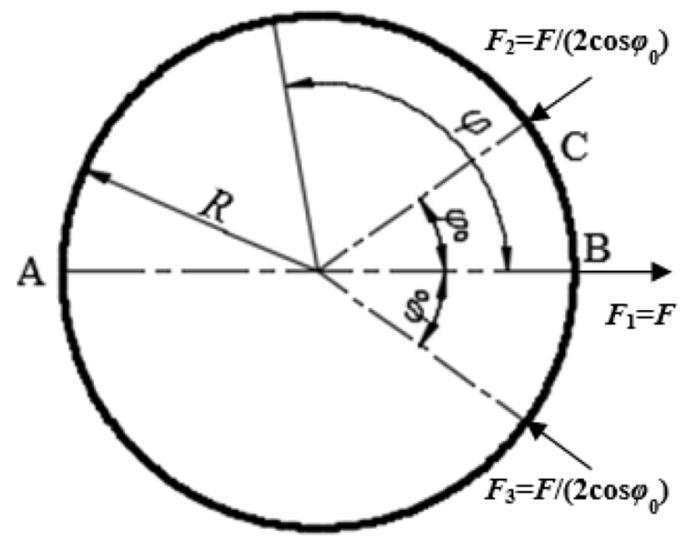

Fig. 3. Structure diagram of the ring under the action of three forces.

\subsection{Distributed bending moment $M_{\mathrm{AB}}(\varphi)$ on ring}

The structure diagram of the three-force ring model is shown in Figure 3. If $F_{1}=F$ is supposed, then other two radial forces $F_{2}$ and $F_{3}$ can be known according to the equilibrium relationship of the force system. The ring is cut along the horizontal midline and the upper part of the ring is taken for analysis, as shown in Figure 4.

When the bending moment $M_{\mathrm{A}}, M_{\mathrm{B}}$ and radial force system act respectively on the half ring shown in Figure 4, the normal angles of section A and B could be calculated by the unit force method [17]. Then the total normal angles of two sections are obtained by superposition. According to deformation compatibility condition, the values of these two normal angles are 0 , so the bending moments are given by:

$$
\begin{aligned}
& M_{A}=F R\left(h_{2}-3 h_{1}\right) /(8 \pi) \\
& M_{B}=F R\left(h_{1}-3 h_{2}\right) /(8 \pi)
\end{aligned}
$$

where $h_{1}$ and $h_{2}$ are given by:

$h_{1}=2-2 \cos \varphi_{0}+\varphi_{0} \tan \varphi_{0}-2 \sin \varphi_{0} \tan \varphi_{0}+\pi \tan \varphi_{0} / 2$
$h_{2}=2-2 \cos \varphi_{0}-\varphi_{0} \tan \varphi_{0}-2 \sin \varphi_{0} \tan \varphi_{0}+3 \pi \tan \varphi_{0} / 2$

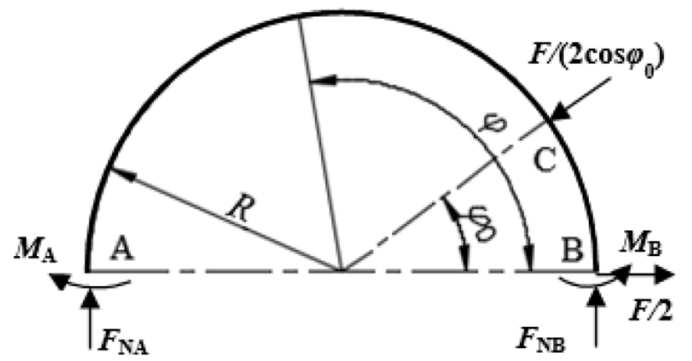

Fig. 4. Internal force of the symmetric section of the ring.

In the same way, when the bending moment $M_{\mathrm{A}}, M_{\mathrm{B}}$ and radial force system act together on the half ring, the total distributed bending moment on ring could be calculated by superposition. It is given by:

$$
\begin{array}{ll}
M_{B C}(\varphi)=F R\left(h_{3}-h_{4} \cos \varphi-\sin \varphi\right) / 2 & 0 \leq \varphi \leq \varphi_{0} \\
M_{A C}(\varphi)=F R\left(h_{3}-h_{5} \cos \varphi\right) / 2 & \varphi_{0} \leq \varphi \leq \pi
\end{array}
$$

where $h_{3}, h_{4}$ and $h_{5}$ are given by:

$$
\begin{aligned}
& h_{3}=\left(h_{1}+h_{2}\right) /(4 \pi)-\tan \varphi_{0} / 2 \\
& h_{4}=\left(h_{1}-h_{2}\right) /(2 \pi)-\tan \varphi_{0} / 2 \\
& h_{5}=\left(h_{1}-h_{2}\right) /(2 \pi)+\tan \varphi_{0} / 2
\end{aligned}
$$

\subsection{The expression of radial displacement}

Substituting equation (6) into equation (1), differential equations are given by:

$$
\begin{array}{ll}
\frac{d^{2} w_{B C}}{d \varphi^{2}}+w_{B C}=-\frac{F R^{3}}{2 E I}\left(h_{3}-h_{4} \cos \varphi-\sin \varphi\right) & 0 \leq \varphi \leq \varphi_{0} \\
\frac{d^{2} w_{A C}}{d \varphi^{2}}+w_{A C}=-\frac{F R^{3}}{2 E I}\left(h_{3}-h_{5} \cos \varphi\right) & \varphi_{0} \leq \varphi \leq \pi
\end{array}
$$


The general solution of two differential equations is expressed as:

$$
\begin{aligned}
& w_{B C}=A_{1} \sin \varphi+A_{2} \cos \varphi-\frac{F R^{3}}{2 E I} \\
& \times\left(h_{3}-\frac{1}{2} h_{4} \varphi \sin \varphi+\frac{1}{2} \varphi \cos \varphi\right) \quad 0 \leq \varphi \leq \varphi_{0} \\
& w_{A C}=B_{1} \sin \varphi+B_{2} \cos \varphi-\frac{F R^{3}}{2 E I} \\
& \quad \times\left(h_{3}-\frac{1}{2} h_{5} \varphi \sin \varphi\right) \quad \varphi_{0} \leq \varphi \leq \pi
\end{aligned}
$$

where $A_{1}, A_{2}, B_{1}$ and $B_{2}$ are integral constants.

Because of the symmetry of structure, the normal angle $\theta$ and the circumferential displacement $v$ equal to 0 when $\varphi=0$ and $\varphi=\pi$. Substituting this symmetric condition into equation (3), $A_{1}$ and $B_{1}$ are given by:

$$
A_{1}=\frac{F R^{3}}{4 E I}, B_{1}=-\frac{F R^{3}}{4 E I} \varphi_{0} \tan \varphi_{0}
$$

Then according to the condition of smooth connection, that is when $\varphi=\varphi_{0}$, equations (9) and (10) should get the same values and derivatives. The same result is given by:

$$
B_{2}=\left(A_{1}-B_{1}\right) \tan \varphi_{0}+A_{2}-\frac{F R^{3} \varphi_{0}}{4 E I \cos ^{2} \varphi_{0}}
$$

It seems that the above conditions are not enough to get a solution, this is because of the lack of horizontal constraints for the structure in Figure 3. To completely determine the four integration constants, we let the radial displacement at point $\mathrm{B}$ equal to $x_{0}$ in Figure 3 . According to equations (9), (11) and (12), $A_{2}$ and $B_{2}$ denoted by $x_{0}$ can be given by:

$$
A_{2}=\frac{F R^{3}}{2 E I} h_{3}+x_{0}, B_{2}=\frac{F R^{3}}{2 E I}\left(h_{3}+\frac{1}{2} \tan \varphi_{0}-\frac{1}{2} \varphi_{0}\right)+x_{0}
$$

The expression of $x_{0}$ needs to be defined. Comparing the mechanical models of two-force ring and three-force ring, the circumferential displacement at $\varphi=\pi / 2$ on the ring should be 0 to ensure that the displacement of point $B$ relative to the vertical line passing through the center of the circle is constant. And through equation (2), the circumferential displacement of the ring can be obtained by

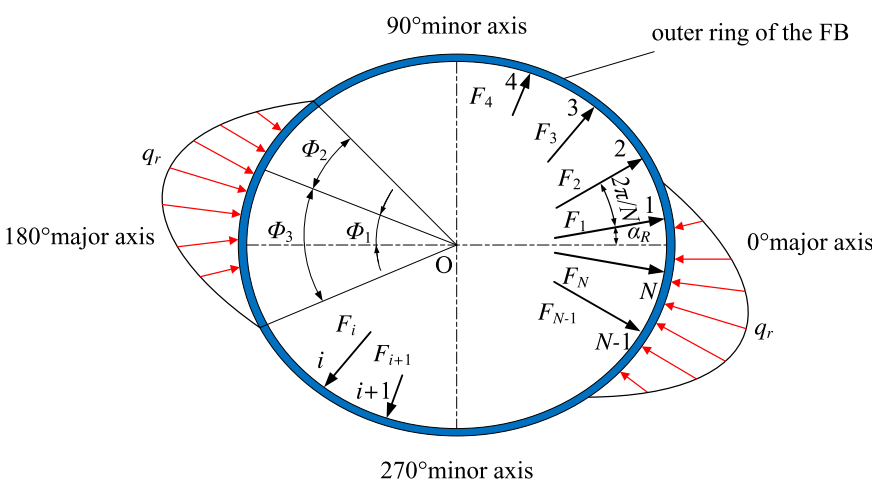

Fig. 5. Loading diagram of outer ring of the FB with $N$ balls $(N \geqq 5)$.

integrating the radial displacement. By integral calculation, the expression of $x_{0}$ is given by:

$$
x_{0}=\frac{F R^{3}}{2 E I}\left[\frac{1}{2}\left(\varphi_{0}-\tan \varphi_{0}-h_{5}\right)-\left(\frac{\pi}{2}+1\right) h_{3}\right]
$$

Finally, substituting equations (11), (13) and (14) into equations (9) and (10), the radial displacement of three-force ring can be given by:

$$
w_{A B}=F H(\varphi) \quad 0 \leq \varphi \leq \pi
$$

where $\mathrm{H}(\varphi)$ is the coefficient of bending deformation, which is given by:

$$
\text { See equation (16) below. }
$$

\section{Load distribution in the flexible bearing}

\subsection{Calculation parameters}

When the FS is loaded, the loading diagram of outer ring of the FB with $N$ balls $(N \geqq 5)$ is shown in Figure 5 . For the simplification of calculation, we have the following assumptions: (1) It is assumed that there is no friction between the CS and the FS. (2)It is assumed that the rotational speed of the shaft has no influence on the radial force state of the FB. (3) It is assumed that the centrifugal load of the balls on the outer ring is much smaller than the ball load itself, which can be ignored. The parameters related to external radial distributed load are given in Table 1, and the main geometric parameters of flexible bearing are given in Table 2 . The values of $\Phi_{1}, \Phi_{2}$ and $\Phi_{3}$ determine the location of the radial distributed load. In this

$$
H(\varphi)= \begin{cases}\frac{R^{3}}{2 E I}\left[\frac{1}{2}\left(\sin \varphi+h_{4} \varphi \sin \varphi-\varphi \cos \varphi\right)+\frac{1}{2}\left(\varphi_{0}-\tan \varphi_{0}-h_{5}-\pi h_{3}\right) \cos \varphi-h_{3}\right] \quad 0 \leq \varphi \leq \varphi_{0} \\ \frac{R^{3}}{2 E I}\left[-\frac{1}{2} \varphi_{0} \tan \varphi_{0} \sin \varphi-\frac{1}{2}\left(h_{5}+\pi h_{3}\right) \cos \varphi+\frac{1}{2} h_{5} \varphi \sin \varphi-h_{3}\right] & \varphi_{0} \leq \varphi \leq \pi\end{cases}
$$


Table 1. Relevant Parameters of radial distributed load.

\begin{tabular}{ll}
\hline Parameter & Value \\
\hline Profile angle $\tau$ & $20^{\circ}$ \\
Pitch diameter of flexspline $d_{r}$ & $50.8 \mathrm{~mm}$ \\
Working width of gear ring $b_{w}$ & $7.2 \mathrm{~mm}$ \\
$\Phi_{1}$ & $0 \mathrm{rad}$ \\
$\Phi_{2}, \Phi_{3}$ & $\pi / 3 \mathrm{rad}$ \\
Load torque $T$ & $0-100 \mathrm{Nm}$ \\
\hline
\end{tabular}

paper, the values of these three variables refer to a relevant paper [18]. The main variables are the load torque and the number of balls, which can be changed in a certain range.

\subsection{The deformation compatibility equation}

In this paper, the cosine-cam is used for analysis of internal load of the FB. The ideal pre-deformation caused by this kind of cam is given by:

$$
w_{\text {cam }}=w_{0} \cos (2 \varphi)
$$

when $\Phi_{2}=\Phi_{3}$, the radial distributed load in Figure 5 is given by $[19,20]$ :

$$
\begin{gathered}
q_{r}=q_{t \max } \tan \tau \cos \left[\left(\phi-\Phi_{1}\right) \pi /\left(2 \Phi_{2}\right)\right] \\
q_{t \max }=\pi T /\left(2 \Phi_{2} d_{r}^{2} b_{w}\right)
\end{gathered}
$$

where $q_{\text {tmax }}$ is the maximal value of tangential distributed load; $\tau$ is profile angle; $d_{r}$ is the pitch diameter of the FS; $b_{w}$ is the working width of gear ring.

Under the radial distributed load, the radial deformation of the outer ring is given by [15]:

$$
\begin{aligned}
& w_{q}=B_{o} R^{4} / E I \sum_{k=2,4,6 \ldots} q_{r k} /\left(k^{2}-1\right)^{2} \cos \left[k\left(\phi-\Phi_{1}\right)\right] \\
& q_{r k}=8 \Phi_{2} q_{t \max } \cos \left(k \Phi_{2}\right) \tan \tau /\left(\pi^{2}-4 k^{2} \Phi_{2}^{2}\right)
\end{aligned}
$$

where $B_{\mathrm{o}}$ is the width of outer ring; $R$ is the neutral layer radius of outer ring; $q_{r k}$ is the coefficient in the $k$ term of series.

In Figure 5, the radial loads on the steel balls are expressed by $F_{i}$ and each loading point is marked with a number in an anti-clockwise direction. And the angular position of the ith loading point is given by:

$$
\varphi_{i}=2 \pi(i-1) / N+\alpha_{R}, \quad i=1,2, \ldots, N
$$

where $\alpha_{\mathrm{R}}$ is the rotation angle of balls which determines the angular positions of loading points.
Table 2. Main parameters of the FB.

\begin{tabular}{ll}
\hline Parameter & Value \\
\hline Outer diameter of outer ring $D_{o}$ & $49.08 \mathrm{~mm}$ \\
Ditch bottom diameter of outer ring $d_{o}$ & $47.28 \mathrm{~mm}$ \\
Radius of groove curvity of outer ring $r_{o}$ & $2.60 \mathrm{~mm}$ \\
Inner diameter of outer ring $D_{1}$ & $46.82 \mathrm{~mm}$ \\
Width of outer ring $B_{o}$ & $7.2 \mathrm{~mm}$ \\
Inner diameter of inner ring $D_{i}$ & $35.58 \mathrm{~mm}$ \\
Ditch bottom diameter of inner ring $d_{i}$ & $37.74 \mathrm{~mm}$ \\
Radius of groove curvity of inner ring $r_{i}$ & $2.48 \mathrm{~mm}$ \\
Outer diameter of inner ring $D_{2}$ & $38.21 \mathrm{~mm}$ \\
Width of inner ring $B_{i}$ & $8.1 \mathrm{~mm}$ \\
Diameter of ball $D_{w}$ & $4.77 \mathrm{~mm}$ \\
Number of balls $N$ & $5-25$ \\
Inertial moment of outer ring section $I$ & $0.7875 \mathrm{~mm}{ }^{4}$ \\
Neutral layer radius of outer ring $R$ & $23.995 \mathrm{~mm}$ \\
Maximal pre-deformation $w_{0}$ & $0.3 \mathrm{~mm}$ \\
(positive on the major axis and & \\
negative on the minor axis) & \\
Elastic modulus $E$ & $207 \mathrm{Gpa}$ \\
Poisson's ratio $\mu$ & 0.3 \\
Radial clearance $P_{d}$ & $0 \mathrm{~mm}$ \\
\hline
\end{tabular}

Then the corresponding deformation compatibility equation can be established at each loading point. The equation of the ith loading point is given by:

$$
w_{i}=w_{\mathrm{cam}, i}-w_{q, i}-P_{d} / 2
$$

where $w_{\mathrm{cam}, i}$ is the pre-deformation caused by cam assembly; $w_{q, i}$ is the deformation caused by the external radial load $q_{r} ; w_{i}$ is the deformation caused by the internal radial load $F_{i} ; P_{d}$ is the radial clearance of the FB.

\subsection{The superposition algorithm of three-force system}

Unknown radial loads $F_{i}(\mathrm{i}=1,2,3, \ldots, N, N \geqq 5)$ shown in Figure 5 can be decomposed into $N$ unknown three-force systems. Each force system is shown in Figure 3, in which the radial force in the middle is $X_{i}(\mathrm{i}=1,2,3, \ldots, N, N \geqq 5)$ and its angular position is determined by equation (20). The radial deformation of different force system at each loading point can be obtained by equations (15) and (16). After all the force systems are superimposed, the total radial deformations at $N$ loading points can be expressed by matrix:

See equation (22) below.

$$
\left[\begin{array}{c}
w_{1} \\
w_{2} \\
\vdots \\
w_{N-1} \\
w_{N}
\end{array}\right]=\left[\begin{array}{ccccc}
H(0) & H\left(\varphi_{0}\right) & \ldots & H\left((N-2) \varphi_{0}\right) & H\left((N-1) \varphi_{0}\right) \\
H\left(\varphi_{0}\right) & H(0) & H\left(\varphi_{0}\right) & \cdots & H\left((N-2) \varphi_{0}\right) \\
\vdots & \vdots & \ddots & \vdots & \vdots \\
H\left((N-2) \varphi_{0}\right) & \cdots & H\left(\varphi_{0}\right) & H(0) & H\left(\varphi_{0}\right) \\
H\left((N-1) \varphi_{0}\right) & H\left((N-2) \varphi_{0}\right) & \cdots & H\left(\varphi_{0}\right) & H(0)
\end{array}\right] \times\left[\begin{array}{c}
X_{1} \\
X_{2} \\
\vdots \\
X_{N-1} \\
X_{N}
\end{array}\right]
$$


where $\varphi_{0}=2 \pi / N$. The above matrix can be simplified as:

$$
[w]=[A][X]
$$

where $[w]$ is the column matrix of radial deformation of outer ring; $[A]$ is the deformation coefficient matrix; $[X]$ is the column matrix of unknown forces.

The deformations at loading points in column matrix $[w]$ can be calculated by equation (21), then we can get a set of linear equations. By solving the equations, $[X]$ is given by:

$$
[X]=[A]^{-1}[w]
$$

The unknown radial loads in Figure 4 can be calculated by superposing unknown forces $X_{i}$ at each loading point. The superposition process is written in the form of matrix, which is given by:

$$
\left[\begin{array}{c}
F_{1} \\
F_{2} \\
\vdots \\
\vdots \\
F_{N-1} \\
F_{N}
\end{array}\right]=\left[\begin{array}{cccccc}
1 & c & 0 & \cdots & 0 & c \\
c & 1 & c & 0 & \cdots & 0 \\
0 & c & 1 & \ddots & \ddots & \vdots \\
\vdots & 0 & c & \ddots & c & 0 \\
0 & \vdots & \ddots & \ddots & 1 & c \\
c & 0 & \cdots & 0 & c & 1
\end{array}\right] \times\left[\begin{array}{c}
X_{1} \\
X_{2} \\
\vdots \\
\vdots \\
X_{N-1} \\
X_{N}
\end{array}\right]
$$

where $c=-1 /\left(2 \cos \varphi_{0}\right)$. The above matrix can be simplified as:

$$
[F]=[C][X]
$$

where $[F]$ is the column matrix of radial loads; $[C]$ is the transformation matrix.

According to the above linear algorithm, the internal radial load of the FB can be calculated. If there are negative results, it indicates that these steel balls are not bearing load. In order to eliminate the negative load, the force boundary condition $F_{i}=0$ should be applied for the position where the ball is not under load. The above calculations can be programmed, and the diagram is shown in Figure 6.

And it should be noted that the superposition algorithm of three-force system is not suitable for the case that the number of balls is less than 5 . When there are three balls, the radial load couldn't be determined because the radial deformations at loading points are the same by equations (15) and (16), it is impossible to establish the linear equation. And when there are two or four balls, the threeforce system is not balanced, but the radial load can be calculated by two-force ring model.

\subsection{The verification of the flexible bearing analytic model}

In order to compare the load distribution results with Xiong's results, the main parameters of bearing LY-6025 in that paper are taken to the above model [12]. The ball load distribution curves with no external loads are shown in

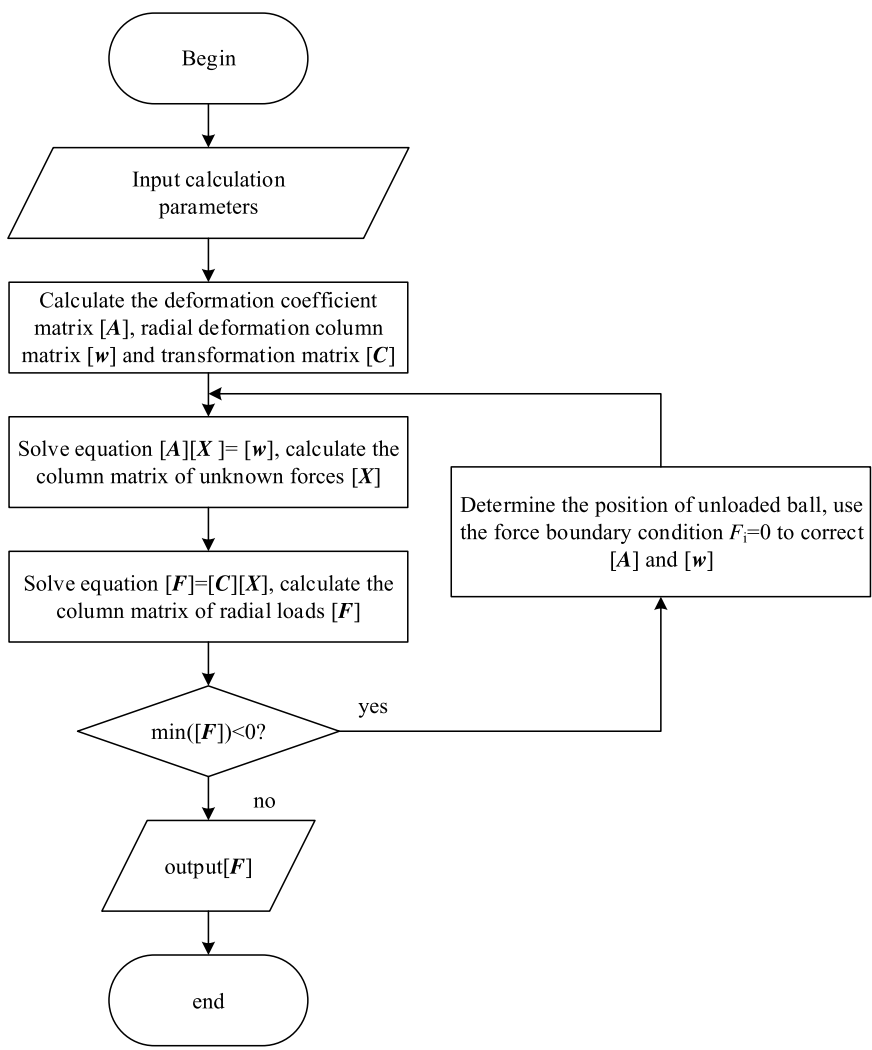

Fig. 6. The block diagram of load distribution calculations for the FB.

Figure 7 . The line with squares represents the ball load distribution result calculated by the three-force ring superposition method, while the line with dots represents that by the static analysis model and the line with triangles represents that by the FEM simulation model in Xiong's paper [12]. It can be seen from the figure that the result of three-force ring superposition method and is similar to that of the compared model. The maximal error between two numerical solutions is within $10 \%$, which occurs around the major axis. And both of the number of no-load balls is 8 . The reason for the differences may be the hertz contact deformation and the nonlinear part in the compared model. Therefore, the correctness and validity of the three-force ring superposition model are proved.

\subsection{The characteristics of load distribution}

\subsubsection{The influence of number of balls on maximum ball} load

In order to obtain the characteristics of load distribution, we took maximum ball load as study object, similar results are obtained for other ball loads. The relevant calculation parameters are listed in Tables 1 and 2 .

According to the algorithm in 3.3, the relationship of maximum ball load and number of balls under load is derived, as shown in Figure 8. It can be seen from figure that the maximum ball load under different load torque always decreases with the increase of number of balls. 


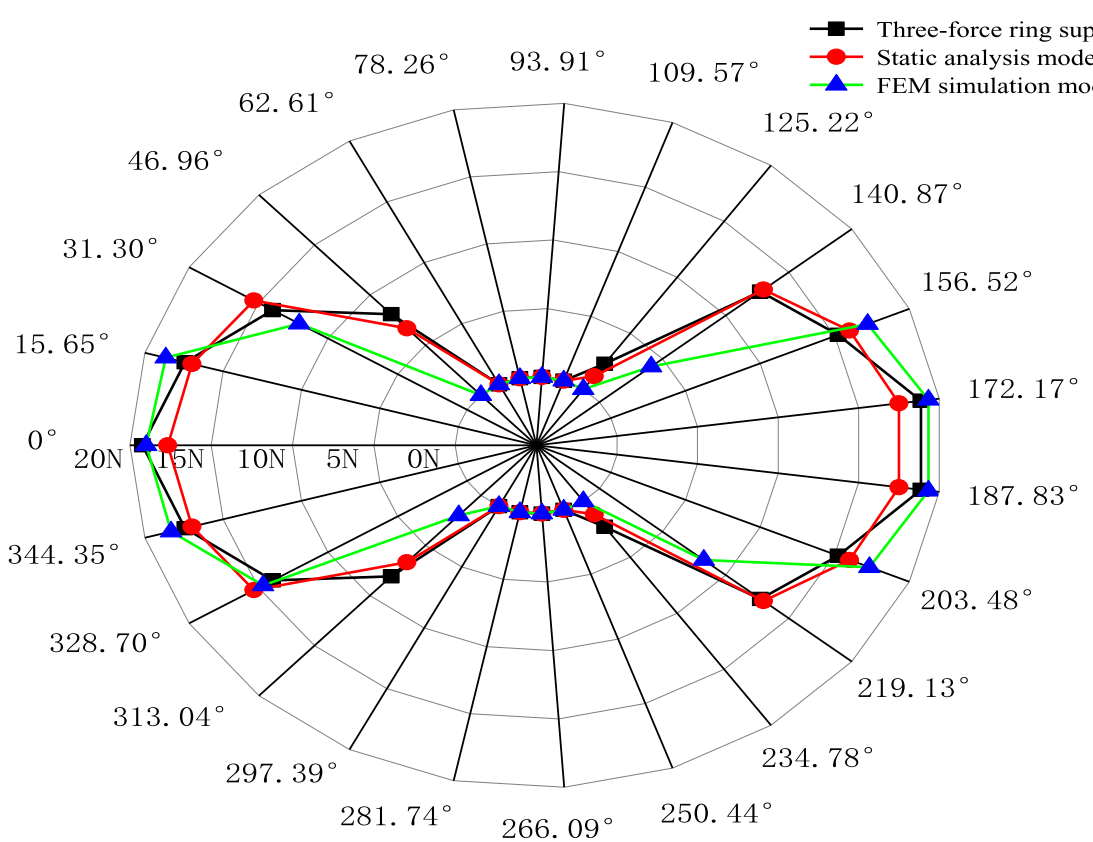

Fig. 7. The ball load distribution comparison of the FB LY-6025.

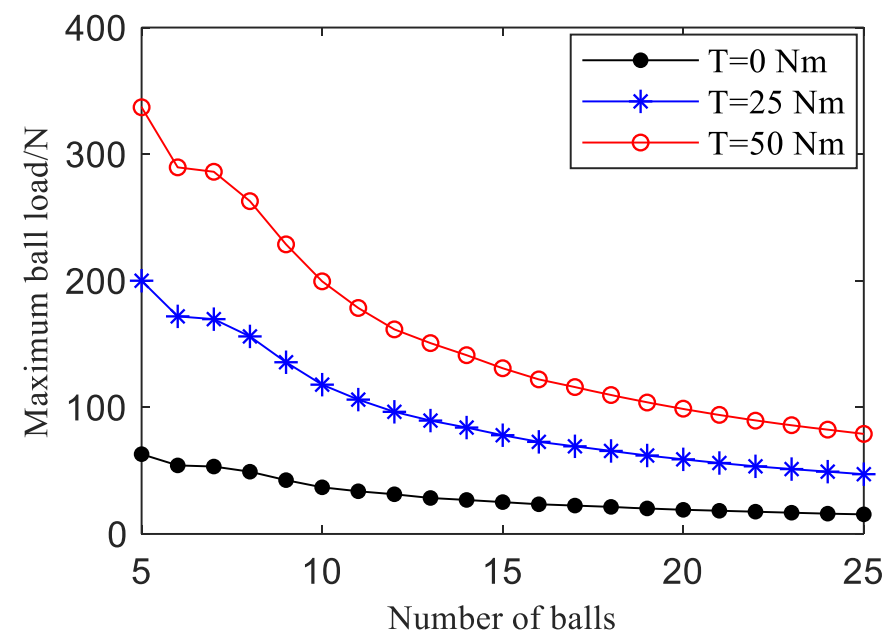

Fig. 8. Relationship of maximum ball load and number of balls under load.

\subsubsection{The influence of load torque on maximum ball load}

When $\alpha_{\mathrm{R}}=0$, the relationship of maximum ball load and load torque under different number of balls is derived, as shown in Figure 9. It can be seen from figure that the maximum ball load increases linearly with the increase of the load torque. And the larger the number of balls, the smaller the slope of the line. This explains that when load torque is large, the maximum ball load of the FB with more balls is far less than that of the FB with less balls.

\subsubsection{The relationship of ball load and angular position}

When the number of balls is 23 , the continuous load curves under different load torque can be obtained by changing the rotation angle of balls, as shown in Figure 10. It can be

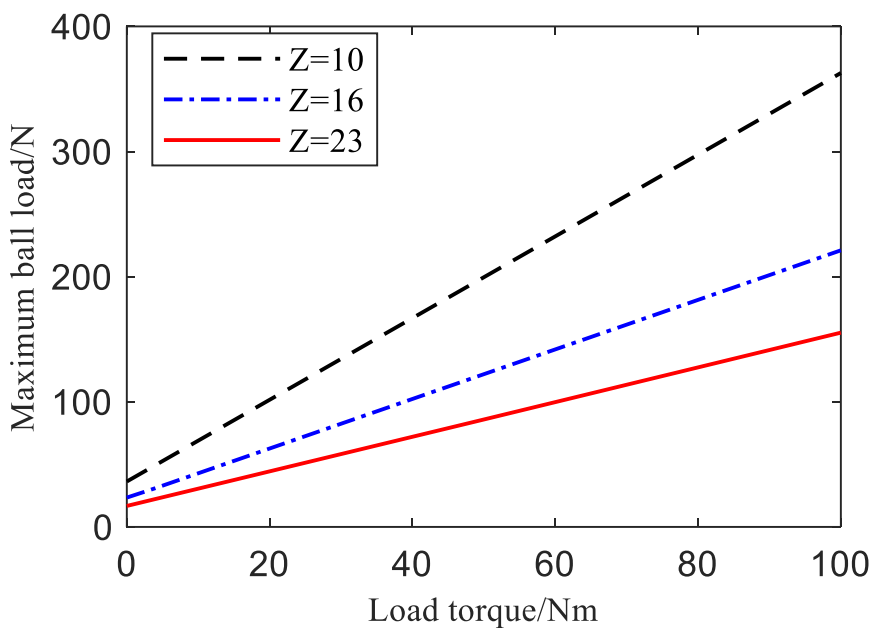

Fig. 9. Relationship of maximum ball load and load torque under different number of balls.

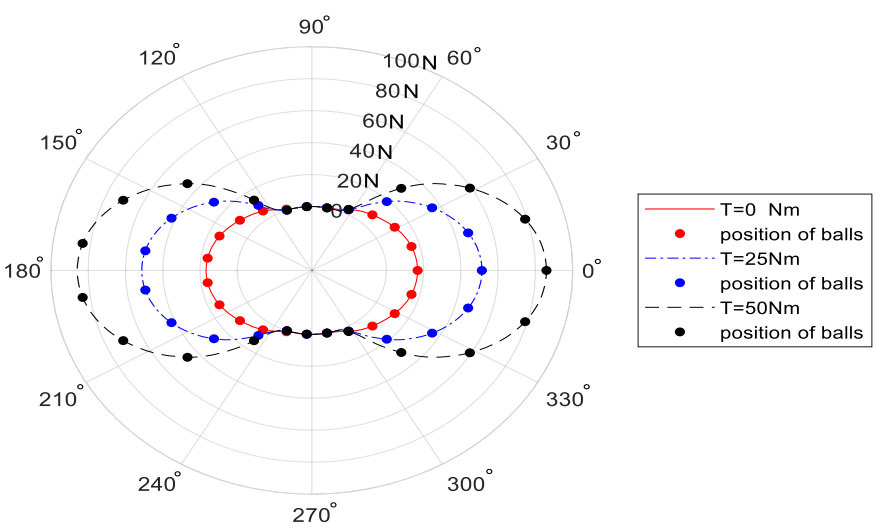

Fig. 10. Relationship of ball load and angular position under load. 
seen from figure that the amplitude of ball load is increased significantly by load torque. When $T=0$, the width of no-load area at the end of minor axis is $30.39^{\circ}$; When $T=50 \mathrm{Nm}$, the width of no-load area increases to $44.1^{\circ}$. Apparently, the load torque narrows the load range of the flexible bearing, resulting in the reduction of the number of loaded balls. For the FB with 23 balls, the number of loaded balls decreases from 19 to 17 after the load torque increases from 0 to $50 \mathrm{Nm}$. Thus, high load torque on the FB should be avoided, which may cause the noise and the abnormal operation of the FB.

\section{The deformation of outer ring of the flexible bearing}

\subsection{The expression of the radial deformation}

An analytical method to account for the structural deformation of the rings was derived [21]. And it assumes that the single load applied to the thin ring is balanced by a symmetric and tangential shear stress distribution, which is not suitable for this paper. In the former two chapters, the relationship between radial deformation and load of three-force ring was derived and the unknown loads were decomposed into several three-force systems. During the above process, the sizes and positions of these three-force systems were derived. Therefore, the total radial deformation of all points on the ring could be calculated by superimposing the radial deformation caused by each three-force system. For the FB with $N$ balls, the radial loads on the outer ring can be decomposed into $N$ threeforce systems, and $X_{\mathrm{i}}$ represents the size of the ith force system. According to the equations (15), (16) and (20), the radial deformation caused by the ith force system is given by:

$$
w_{i}(\varphi)=X_{i} H\left(\beta_{i}\right)
$$

where $\beta_{\mathrm{i}}$ is given by:

$$
\beta_{i}= \begin{cases}\left|\varphi_{i}-\varphi\right| & \text { 当 }\left|\varphi_{i}-\varphi\right| \leq \pi \\ 2 \pi-\left|\varphi_{i}-\varphi\right| & \text { 当 }\left|\varphi_{i}-\varphi\right|>\pi\end{cases}
$$

Therefore, the total radial deformation of outer ring under $N$ three-force systems and radial distributed load $q_{r}$ is given by:

$$
w(\varphi)=w_{q}+\sum_{i=1}^{N} X_{i} H\left(\beta_{i}\right)
$$

where $w_{\mathrm{q}}$ is determined by equation (19).

\subsection{The characteristics of the radial deformation of outer ring}

4.2.1 The influence of number of balls on the radial deformation of outer ring

With the increase of the number of balls, the theoretical deformation will be closer to the ideal pre-deformation. In order to study the influence of number of balls on radial

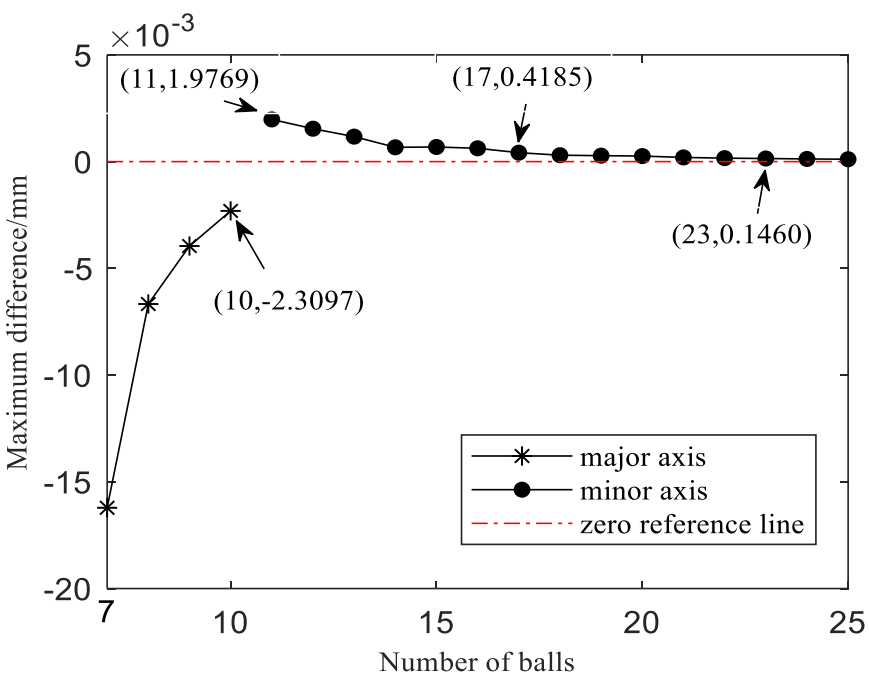

Fig. 11. Relationship of the maximum difference and number of balls under no-load.

deformation, the difference of the expectation and the theoretical result is calculated, which is given by:

$$
\Delta w=w(\varphi)-w_{\text {cam }}(\varphi)
$$

where $w(\varphi)$ is the theoretical radial deformation, which can be calculated by equation $(29) ; w_{\text {cam }}(\varphi)$ is the ideal radial deformation, which is determined by equation (17).

When $\alpha_{R}=0$ and $T=0$, the relationship curve of the maximum difference $\Delta w_{\max }$ and the number of balls $N$ is obtained, shown in Figure 11. It can be seen from figure that the maximum difference is negative and it appears at the end of the major axis when $N \leq 10$. This will have an adverse effect on the engaging state of teeth here. And when $N>10$, the maximum difference is positive and it appears at the end of the minor axis. Overall, the maximum difference decreases with the increase of the number of balls.

\subsubsection{The influence of load torque on the radial} deformation of outer ring

When $N=23$ and $\alpha_{R}=0$, the relationship curve of the difference of radial deformation $\Delta w$ and angular position $\varphi$ under $T=0$ and $T=50 \mathrm{Nm}$ is obtained, as shown in Figure 12. In the figure, the dash dotted line represents the theoretical deformation shape when $T=0$ and the solid line represents the theoretical deformation shape when $T=50 \mathrm{Nm}$. And the expected deformation shape is indicated by the dotted line, which represents zero position. And the dots indicate the position of the balls. It can be seen from figure that the amplitude of $\Delta w$ can be increased by load torque. When $T=50 \mathrm{Nm}$, the maximum difference at the end of minor axis is 2.3 times of that under no-load. And the deformation shape is actually wavelike. The theoretical curve coincides with the expected curve at the loading points, and the shapes are similar around these points. But there is an obvious difference between the theoretical and expected deformation shape around the minor axis. And 


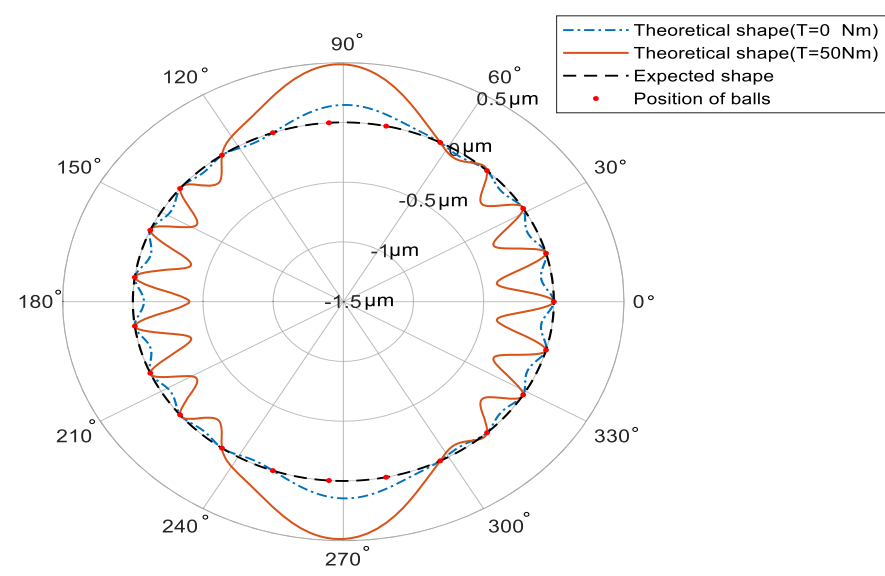

Fig. 12. Relationship of the difference of radial deformation and angular position under load.

the shape is convex, which results in no-load balls. If the radial deformation here is too large, it may cause that the teeth around the minor axis of the FS mesh with the teeth of steel wheel, which should be avoided in harmonic drive. And it can be found that the number of no-load balls will increase under load torque, which is consistent with the result in 3.5.3.

\section{The bending normal stress of outer ring of flexible bearing}

\subsection{The bending normal stress distribution on the outer ring}

The method for calculating the bending moment caused by ball load is similar to the method in 4.1. According to equations (6) and (7), the bending moment at any section of outer ring $M_{F}(\varphi)$ can be calculated by superimposing the bending moment caused by each three-force system, which is given by:

$$
M_{F}(\varphi)=\sum_{i=1}^{n} X_{i} G_{M}\left(\beta_{i}\right)
$$

where $G_{M}(\varphi)$ is given by:

$$
G_{M}(\varphi)= \begin{cases}R\left(h_{3}-h_{4} \cos \varphi-\sin \varphi\right) / 2 & 0 \leq \varphi \leq \varphi_{0} \\ R\left(h_{3}-h_{5} \cos \varphi\right) / 2 & \varphi_{0} \leq \varphi \leq \pi\end{cases}
$$

Substituting equation (19) into equation (1), the bending moment caused by radial distributed load is given by:

$$
M_{q}(\varphi)=B_{o} R^{2} \sum_{k=2,4,6 \ldots} q_{r k} /\left(k^{2}-1\right) \cos \left[k\left(\varphi-\varphi_{1}\right)\right]
$$

Consequently, the total bending moment on the outer ring is given by:

$$
M(\varphi)=M_{F}(\varphi)+M_{q}(\varphi)
$$

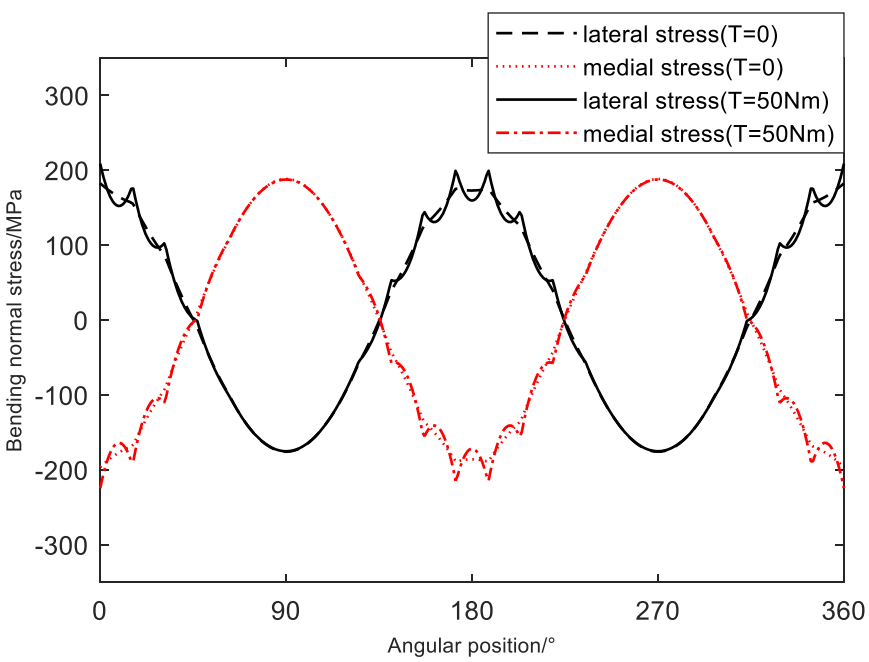

Fig. 13. Bending normal stress distribution of outer ring of the FB.

Considering the small wall thickness of outer ring, the bending normal stress of outer ring can be calculated by bending theory of straight beam [22], whose expression is given by:

$$
\sigma_{M}=M(\varphi)(r-R) / I
$$

where $r$ is the radius of anywhere on cross section of outer ring; $R$ is the neutral layer radius of outer ring; $I$ is the inertial moment on cross section.

When $N=23$ and $\alpha_{R}=0$, the bending normal stress of outer ring under no load can be calculated by equation (35), the results are shown in Figure 13. The stress curves are smooth under no-load condition. For the bending normal stress outside the cross section of outer ring, the maximum tensile stress which appears at the end of major axis is 182.7 MPa, and the maximum compressive stress which appears at the end of minor axis is $-171.6 \mathrm{MPa}$. And the distribution of the bending normal stress inside the cross section of outer ring is contrary.

When load torque $T=50 \mathrm{Nm}$, the curve of the bending normal stress distribution changes. It can be seen from figure that load torque will cause obvious extreme points on the stress curve. And the maximum bending normal stress at the end of the major axis increases apparently, but the maximum bending stress at the end of the minor axis barely change.

\subsection{The characteristics of maximum bending normal stress}

5.2.1 The influence of number of balls on the maximum bending normal stress

The Figure 14 shows the relationship between the maximum bending normal stress of lateral and medial outer ring and the number of balls under no load. The lateral stress represents the maximum bending normal stress outside the cross section of outer ring, and the medial stress represents that inside the cross section of outer ring. 


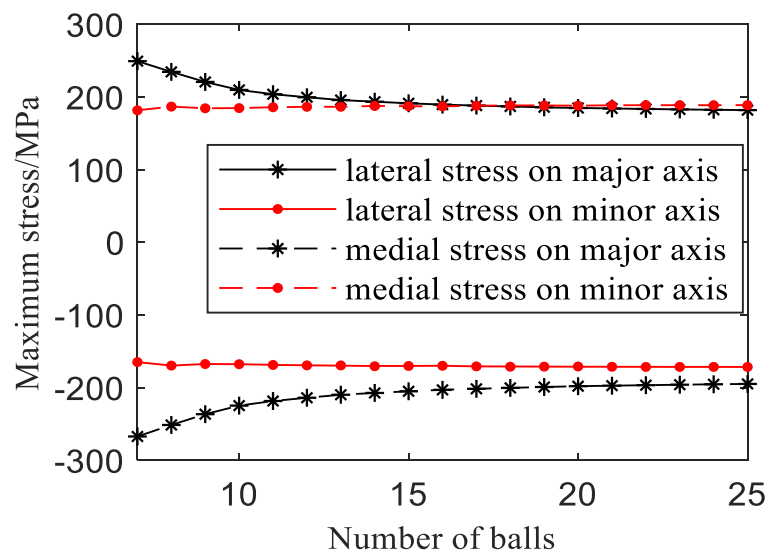

Fig. 14. The influence of number of balls on the maximum bending normal stress.

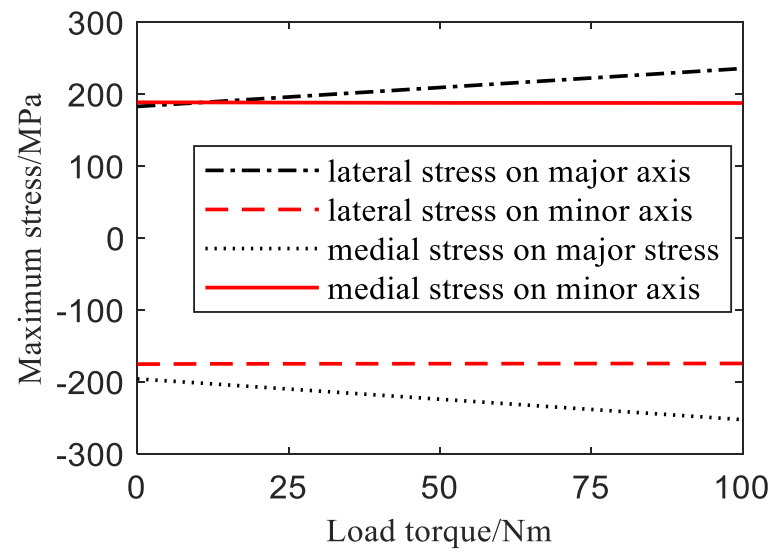

Fig. 15. The influence of load torque on the maximum bending normal stress.

It can be found that the lateral and medial stress have the same trend, that is the stress on major axis decreases with the increase of number of balls but the stress on minor axis stays the same. For example, looking into the curves of the lateral stress, we can find that the maximum tensile stress decreases from 249.1 to $181.8 \mathrm{MPa}$ after the number of balls increases from 7 to 25 , which is reduced by $27 \%$. And the maximum compressive stress on minor axis is maintained at about $170 \mathrm{MPa}$.

\subsubsection{The influence of load torque on the maximum bending normal stress}

The relationship between the maximum bending normal stress of lateral and medial outer ring and load torque is shown in Figure 15. It can be found that the bending normal stress on major axis increases with the increase of load torque but the bending normal stress on minor axis stays the same. For example, looking into the curves of the lateral stress, we can find that the maximum tensile stress increases from 182.7 to $235.5 \mathrm{MPa}$ after the load torque increases from 0 to $100 \mathrm{Nm}$, which is augmented by $28.9 \%$. And the maximum compressive stress on minor axis is maintained at about $-175 \mathrm{MPa}$.

\section{Conclusions}

A load analysis model of three-force ring for the FB was proposed in this paper and the main conclusions were as follows:

- Based on the theory of thin-walled ring, a mechanical model of three-force ring for the FB was established to derive the relationship between radial deformation and load of the outer ring. And then the load distribution in the FB was obtained by superposition of three-force ring. This superposition algorithm of three-force ring is suitable for the case that the number of balls is more than 4. And the calculation speed of this linear algorithm is much higher than FEMs.

- According to the superposition algorithm of three-force ring, the influence of number of balls, load torque and angular position on the maximum ball load was studied.

The results show that the maximum ball load always increases with the increase of number of balls and it increases linearly with the increase of the load torque. For the FB with 23 balls, the width of no-load area at the end of minor axis increases from $30.39^{\circ}$ to $44.1^{\circ}$ after the load torque increases from 0 to $50 \mathrm{Nm}$. Consequently, the load torque narrows the load range of the $\mathrm{FB}$, resulting in the reduction of the number of loaded balls. Thus, the load torque should be limited to avoid the noise and abnormal operation of the FB.

- The theoretical deformation of outer ring of the FB is closer to the expected deformation with the increase of the number of balls. The load torque will increase the difference of the expectation and the theoretical radial deformation.

The deformation shape is actually wavelike. The theoretical curve coincides with the expected curve at the loading points, and they are similar in shape around these points. But there is an obvious difference between the theoretical and expected deformation shape around the minor axis. And the shape is convex, which results in noload balls. And it should be noticed that a large radial deformation here should be avoided in harmonic drive.

- The alternating bending normal stress on outer ring of the FB was studied. On the outer side, the maximum tensile stress appears on major axis and the maximum compressive stress appears on minor axis. On the inner side, the maximum tensile stress appears on minor axis and the maximum compressive stress appears on major axis. The radial distributed load produced by load torque will cause obvious extreme points on the stress curve. In addition, the bending normal stress on major axis will decreases with the increase of the number of balls and increases with the increase of the load torque. But the stress on minor axis is hardly affected by these two factors.

\section{Nomenclature}

$\begin{array}{ll}N & \text { Number of balls } \\ R & \text { Neutral radius of outer ring } \\ M & \text { Bending moment }\end{array}$


$T \quad$ Load torque

$X_{i} \quad$ Middle radial force in the ith three-force system

$F_{i} \quad$ The ith radial load on outer ring

$w_{\mathrm{AB}} \quad$ Radial deformation of three-force ring

$\Delta w_{\max }$ Maximum difference of radial deformation

$H(\varphi) \quad$ Coefficient of bending deformation

This project is supported by cooperative foundation of BEIJING CTKM HARMONIC DRIVE CO., LTD and Shanghai university (Grant No: D.71-0109-18-076).

\section{References}

[1] I.E. Lyuminarsky, S.E. Lyuminarsky, E.S. Lyu minarskaya, Increased Durability of Flexible Gear of Harmonic Drive Due to Flexible Bearing Modification, Proceedings of the 4th International Conference on Industrial Engineering, ICIE 2018, Moscow, Russian Federation, May 15-18, Springer, Cham, 2018, pp. 1287-1292

[2] Y.W. Shen, Q.T. Ye, Theory and Design of Harmonic Gear Transmission, Machine Industry Press, Beijing, 1985, Chap. 5

[3] W. Ostapski, Analysis of the stress state in the harmonic drive generator-flexspline system in relation to selected structural parameters and manufacturing deviations, Bull. Polish Acad. Sci. Technol. Sci. 58, 683-698 (2010)

[4] T.A. Harris, M.N. Kotadlas, Rolling Bearing Analysis, Taylor \& Francis, New York, 2006

[5] C. Wan, Analysis Method of Rolling Bearing, China Machine Press, 1987

[6] K.X. Le, Y.W. Shen, Load distribution on the wave generator of harmonic gear transmission, Mech. Sci. Technol. Aerosp. Eng. 34, 38-45 (1990)

[7] F.C. Shao et al., Internal load analysis of flexible rolling bearings for harmonic gear transmission, Mach. Des. Manuf. 1, 34-37 (1991)

[8] K.X. Le, Y.X. Quan, G.R. Zhou et al., A new method for calculating load distribution on harmonic rolling bearing, J. Zhejiang Univ. 28, 396-404 (1994)
[9] L. Li, B.D. You, J.M. Wen et al., Load distribution calculation of flexible ball bearing with elliptical cam wave generator, Abstracts of International Conference on Mechanics, Materials and Structural Engineering, 2016, pp. $14-15$

[10] Y. Jiang, Y.Z. Wang, Q. Tong et al., Theoretical calculation and simulation analysis of internal load of flexible bearing in harmonic drive, Mach. Des. Res. 33, 91-94 (2017)

[11] J.Y. Liu, Y.P. Chiu, Analysis of a thin elastic ring under arbitrary loading, J. Eng. Ind. 96, 870-876 (1974)

[12] Y. Xiong et al., Load analysis of flexible ball bearing in a harmonic reducer, J. Mech. Des. 142, 1-52 (2020)

[13] L. Li, Static and dynamic analysis of flexspline and flexible bearing under the cam with typical profile, M.S. thesis, Harbin Institute of Technology, Harbin, 2016

[14] X.Q. Xiang, W.W. Zhang, X.J. Wang, G.Y. Yu, J. Dong, J. Zhang, Stress characteristics' analysis of flexible bearings at different speeds, Metrol. Meas. Tech. 44, 6-8 (2017)

[15] M.H. Ivanov, Harmonic Gear Drives, Visajas Kola Press, Moscow, 1981

[16] I. Lopatukhin, L. Khaskel' berg, Deformation of thin-walled rings under the action of $\mathrm{n}$ concentrated and distributed forces, Int. J. Adv. Manuf. Technol. 49, 627-634 (2010)

[17] R.C. Hibbeler, Statics and Mechanics of Materials, China Machine Press, 2014

[18] Y. Jiang, Y.Z. Wang, K. Zhao et al., Mechanical characteristics analysis of thin-walled flexible bearing in harmonic reducer, Bearing, 10-14 (2017)

[19] Y.N. Fengdai, Simulation analysis of load and test technology research for flexible bearing, Shanghai University, 2017

[20] A.M. Wang, Y.Z. Wang, K. Zhao et al., Mechanical calculation on the flexible bearing of harmonic driver, IOP Conf. Ser. Mater. Sci. Eng. 490, (2019)

[21] G. Cavallaro, D. Nelias, F. Bon, Analysis of high-speed intershaft cylindrical roller bearing with flexible rings, Tribol. Lubr. Technol. 48, 154-164 (2005)

[22] J.R. Barber, Intermediate mechanics of materials, Mater. Today 10, B104 (2007)

Cite this article as: Y. Yu, E. Zhu, X. Chen, Y. Wang, Load analysis and deformation research of the flexible bearing based on a three-force ring superposition method, Mechanics \& Industry 22, 34 (2021) 